\title{
Comparison of the Effects of Single Doses of Elcatonin and Pregabalin on Oxaliplatin-Induced Cold and Mechanical Allodynia in Rats
}

\author{
Manahito Aoki, Yuki Kurauchi, Asami Mori, Tsutomu Nakahara, Kenji Sakamoto,* and \\ Kunio Ishii \\ Department of Molecular Pharmacology, Kitasato University School of Pharmaceutical Sciences; 5-9-1 Shirokane, \\ Minato-ku, Tokyo 108-8641, Japan.
}

Received September 18, 2013; accepted November 20, 2013

Oxaliplatin frequently causes peripheral neuropathy. Clinical studies have indicated that pregabalin ameliorates oxaliplatin-induced peripheral neuropathy. However, pregabalin frequently causes dizziness and somnolence. We previously reported that elcatonin, a synthetic analog of eel calcitonin, attenuated oxaliplatin-induced cold and mechanical allodynia in rats. The aim of the present study was to compare the anti-allodynic effects of elcatonin and pregabalin in the rats developing the oxaliplatin-induced neuropathy. Male Sprague-Dawley rats were treated with a single dose of oxaliplatin $(6 \mathrm{mg} / \mathrm{kg}$, intraperitoneally (i.p.)) to induce cold and mechanical allodynia. We assessed the effects of subcutaneous elcatonin $(20 \mathrm{U} / \mathrm{kg})$ and oral pregabalin $(30 \mathrm{mg} / \mathrm{kg})$ on cold and mechanical allodynia by cold stimulation $\left(8^{\circ} \mathrm{C}\right)$ to the hind paw of the rats and the von Frey test, respectively. Elcatonin reversed the effects of oxaliplatin-induced cold and mechanical allodynia in rats for a longer time period than pregabalin does. These results suggested that elcatonin might be useful for the clinical treatment of oxaliplatin-induced neuropathy.

Key words oxaliplatin; elcatonin; neuropathy; pregabalin; cold allodynia; mechanical allodynia

Oxaliplatin is a platinum-based anti-cancer drug used in the treatment of advanced metastatic colorectal cancer, ${ }^{1)}$ and is known to induce a specific acute and chronic form of neuropathy. ${ }^{2)}$ There are few reports on the use of drugs to prevent or treat this painful neuropathy in patients. Recently, pregabalin was reported to reduce the severity of oxaliplatin-induced sensory neuropathy in rats ${ }^{3)}$ and patients. ${ }^{4)}$ However, pregabalin frequently causes dizziness and somnolence., ${ }^{4,5)}$

Recently, we have reported that repeated administration of calcitonins, such as elcatonin, a synthetic analog of eel calcitonin, and salmon calcitonin, attenuated oxaliplatin-induced cold and mechanical allodynia in rats. ${ }^{6,7)}$ To apply elcatonin in a clinical setting, it is important to compare anti-allodynic effects of elcatonin and pregabalin on the oxaliplatin-induced neuropathy. In the present study, we compared the efficacies of single doses of elcatonin and pregabalin for inhibiting oxaliplatin-induced allodynia in rats.

\section{MATERIALS AND METHODS}

Animals All experimental procedures in this study were approved by the Committee for Animal Experiments, Kitasato University, and complied with the Guiding Principles for the Care and Use of Laboratory Animals, approved by the Japanese Pharmacological Society. The official number of animal experiment approved by the committee for our university is T11-1. Male Sprague-Dawley rats weighing 150-250g (Japan SLC, Hamamatsu, Japan) were used. The rats were housed 4-6 per cage in a room with controlled temperature $\left(22 \pm 2^{\circ} \mathrm{C}\right)$ and humidity $(55 \% \pm 5 \%)$, and a 12 -h light/dark cycle. The animals were allowed free access to regular rat chow and tap water. The researchers performing the behavioral studies were blinded to the treatment being administered.

Drugs Elcatonin $\left(\right.$ Elcitoni $\left.^{\circledR}\right)$ and pregabalin were kindly gifted by Asahi Kasei Pharma Corporation (Tokyo, Japan).

The authors declare no conflict of interest.
Oxaliplatin (Elplat ${ }^{\circledR}$ ) was purchased from Yakult Co., Ltd., Tokyo, Japan. All other chemicals and materials were purchased from standard sources, and were of reagent grade or higher.

Oxaliplatin-Induced Allodynia Models Following baseline measurements of cold and mechanical sensitivity as described below, rats were treated with a single, intraperitoneal (i.p.) dose of oxaliplatin $(6 \mathrm{mg} / \mathrm{kg}$, dissolved in $5 \%$ glucose solution) or vehicle (5\% glucose solution; control group) ${ }^{3)}$ This was administered on Day -2 , two days prior to treatment with either elcatonin or pregabalin. Elcatonin was administered subcutaneously (s.c., $20 \mathrm{U} / \mathrm{kg}$, dissolved in $0.1 \mathrm{~mm}$ sodium acetate [ $\mathrm{pH} 5.5]$ and $0.02 \%$ bovine serum albumin in saline). The dose of elcatonin was the same as that employed in our previous study. ${ }^{6}$ Pregabalin was administered orally (per os (p.o.), $30 \mathrm{mg} / \mathrm{kg}$ in distilled water) $2 \mathrm{~d}$ after oxaliplatin administration (Day 0). The dose of pregabalin was reported to block the mechanical allodynia in chronic constrictive injury rats, ${ }^{8)}$ and docetaxel-induced cold allodynia ${ }^{9)}$ almost completely.

Cold Stimulation Cold allodynia was assessed using a system that we developed by modifying existing equipment to allow cold stimulation to be applied directly to the hind paw of the rats. ${ }^{6,7)}$ The truncated aluminum cone used to apply cold stimulation was maintained at $8^{\circ} \mathrm{C}$. Each rat was placed in a clear plastic box $(20 \times 17 \times 13 \mathrm{~cm})$ with a wire mesh floor and allowed to habituate. The tip of the cold plate was then placed in contact with the rat's hind paw until it withdrew it. The time taken for the rat to withdraw its hind paw from the cold plate ("withdrawal latency") was used as the nociceptive measure. A cut-off period of $15 \mathrm{~s}$ was used to avoid damage to the paw. Ten trials ( 5 trials per hind paw) were carried out at 1 -min intervals. The mean withdrawal latency of the 10 trials was used for data analysis. To determine the effect of elcatonin and pregabalin on cold allodynia induced by oxaliplatin, these tests were performed before oxaliplatin administration (Day -2); $2 \mathrm{~d}$ after oxaliplatin administration (Day 0, before 
elcatonin or pregabalin administration); $0.5,1,2,4$, and $6 \mathrm{~h}$ after elcatonin or pregabalin administration; and on Days 2 and 3 .

von Frey Test Mechanical allodynia was evaluated as described previously using the von Frey test. ${ }^{6,9)}$ Each rat was placed in a clear plastic box $(20 \times 17 \times 13 \mathrm{~cm})$ with a wire mesh floor and was allowed to habituate. The von Frey filament for $4 \mathrm{~g}$ force (Touch Test Sensory Evaluator Set, Stoelting, Wood Dale, IL, U.S.A.) was applied to the midplantar skin of both the hind paws ( 5 trials for each hind paw, 10 trials per rat). Each application was performed for $6 \mathrm{~s}$ and withdrawal responses were counted. To determine the effect of elcatonin or pregabalin on mechanical allodynia induced by oxaliplatin, these tests were performed before oxaliplatin administration (Day -2); $2 \mathrm{~d}$ after oxaliplatin administration (Day 0, before elcatonin or pregabalin administration); $0.5,1,2,4$, and $6 \mathrm{~h}$ after elcatonin or pregabalin administration; and on Days 2 and 3 .

Statistical Analysis The data represent the means S.E.M. At first, we performed repeated-measures (ANOVA). However, the interaction between time and treatment was statistically significant. Therefore, Tukey-Kramer test was performed among all of the measuring points (not at each time point) to make multiple comparisons, if the result of one-way ANOVA was statistically significant. To compare the duration of the anti-nociceptive effect between the drugs, the mean areas under the withdrawal latency or the number of withdrawal responses versus time curves $(A U C)$ were calculated using the trapezoidal rule, according to the previous report. ${ }^{3)}$ Tukey-Kramer test was performed to compare the $A U C$ s, if the result of one-way ANOVA was statistically significant. Differences were considered to be statistically significant when $p<0.05$.

\section{RESULTS}

Figure 1 shows that prior to oxaliplatin or vehicle administration (Day -2), each treatment group had equivalent hind paw withdrawal latencies in response to cold stimulation. On Day 0 , the rats treated with oxaliplatin had significantly shorter withdrawal latencies than the rats treated with the vehicle $(p<0.05)$, indicating that oxaliplatin induced cold allodynia in rats. Administration of elcatonin following the development of oxaliplatin-induced cold allodynia increased withdrawal latency, which was returned to the level observed in the rats untreated with oxaliplatin by $2 \mathrm{~h}$ after elcatonin administration (Fig. 1A). This effect was present for the remainder of the experimental period, and was still statistically significant on Day $2(p<0.05)$. Administration of pregabalin following the development of oxaliplatin-induced cold allodynia rapidly increased withdrawal latency, which had returned to the level observed in the rats untreated with oxaliplatin by $0.5 \mathrm{~h}$ after pregabalin administration (Fig. 1B). This effect was retained up to $6 \mathrm{~h}$ on Day 0 , but had been lost by Day 1. On Day 1 and later, these animals showed similar withdrawal latencies as shown by those treated with oxaliplatin alone.

Figure $1 \mathrm{C}$ shows the $A U C$ of the time-courses of the effects of elcatonin and pregabalin on cold allodynia, that are shown in Figs. 1A and 1B. In the i.p. oxaliplatin plus s.c. vehicle group and the i.p. oxaliplatin plus p.o. vehicle group, $A U C \mathrm{~s}$ of the time-courses changes of withdrawal latencies were sig- nificantly smaller than those of the animals treated with the vehicle alone (Fig. 1C). Administration of elcatonin following the development of cold allodynia significantly increased the $A U C$ to the level that was almost same as the control groups, which received i.p. vehicle $(p<0.05)$. The administration of pregabalin following the development of cold allodynia also significantly increased the $A U C(p<0.05)$, but it was significantly smaller than that of the group that received i.p. oxaliplatin plus s.c. elcatonin $(p<0.05)$.

Figure 2 shows that prior to oxaliplatin or vehicle administration (Day -2), each treatment group had an equivalent number of withdrawal responses in the von Frey test. Rats treated with oxaliplatin tended to have a higher number of withdrawal responses than the vehicle-injected control rats at time 0 , suggesting that oxaliplatin induced mechanical allodynia in rats. However, the development of mechanical allodynia was not statistically significant except in the oxaliplatin plus pregabalin-injected group. Although no significant change of withdrawal responses was found in the oxaliplatin plus elcatonin-injected rats from $0.5 \mathrm{~h}$ to $6 \mathrm{~h}$ after the administration of the drug, mechanical allodynia tended to develop at time 0 , and to restore to the initial levels $0.5 \mathrm{~h}$ after elcatonin administration and later (Fig. 2A). Administration of pregabalin following the development of mechanical allodynia decreased the number of withdrawal responses within $0.5 \mathrm{~h}$ of pregabalin administration, for up to $6 \mathrm{~h}$ (Fig. 2B), and $6 \mathrm{~h}$ after the administration of the drug and later, these animals showed similar withdrawal responses as shown by those treated with oxaliplatin alone.

Figure $2 \mathrm{C}$ shows the $A U C$ of the time courses of the effects on mechanical allodynia presented in Figs. 2A and B. In the i.p. oxaliplatin plus s.c. vehicle group and the i.p. oxaliplatin plus p.o. vehicle group, $A U C$ s of the time courses changes of withdrawal responses was significantly higher than those of the animals treated with the vehicle alone (Fig. 2C). Administration of elcatonin following the development of mechanical allodynia significantly decreased the $A U C$ to the level that was almost same as the control groups, which received i.p. vehicle $(p<0.05)$. Administration of pregabalin following the development of mechanical allodynia did not significantly decrease the $A U C$ compared to the vehicle-injected control groups.

\section{DISCUSSION}

The present study demonstrated that a single administration of either elcatonin or pregabalin was capable of attenuating oxaliplatin-induced allodynia in rats. Elcatonin almost completely reversed oxaliplatin-induced cold allodynia, and tended to reduce mechanical allodynia. These effects lasted for a longer time than those of a single administration of pregabalin. The anti-allodynic effects of elcatonin observed in the present study were very similar to results obtained by repeated administration of elcatonin ${ }^{6)}$ and salmon calcitonin. ${ }^{7)}$ Ling et al. also reported that pregabalin reversed oxaliplatin-induced cold allodynia in rats. ${ }^{3)}$

It has been reported that elcatonin $(1500 \mathrm{U} / \mathrm{kg})$ did not significantly affect spontaneous locomotor activity in mice, ${ }^{10)}$ suggesting that the dose of elcatonin employed in our previous study and in the present study $(20 \mathrm{U} / \mathrm{kg})$ would not have reduced spontaneous locomotor activity. ${ }^{6}$ Although we did not collect objective data relating to locomotor activity, we ob- 
A

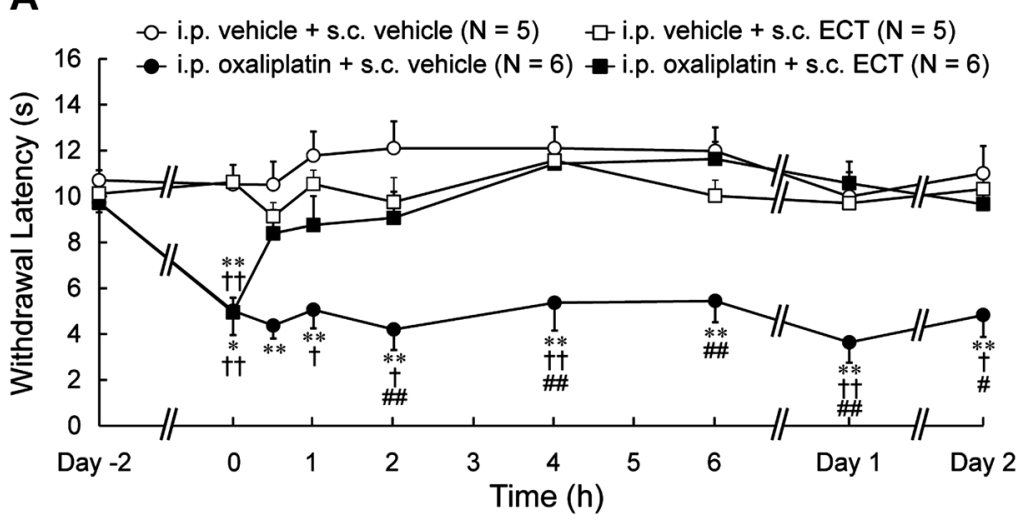

$\mathrm{OX} \uparrow \mathrm{ECT} \uparrow$

B

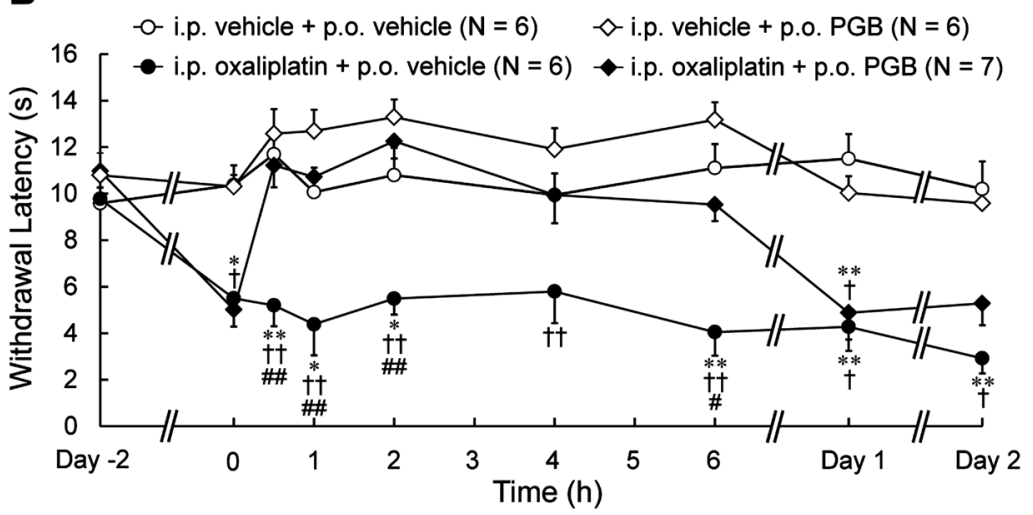

OX $\uparrow \mathrm{PGB} \uparrow$

C

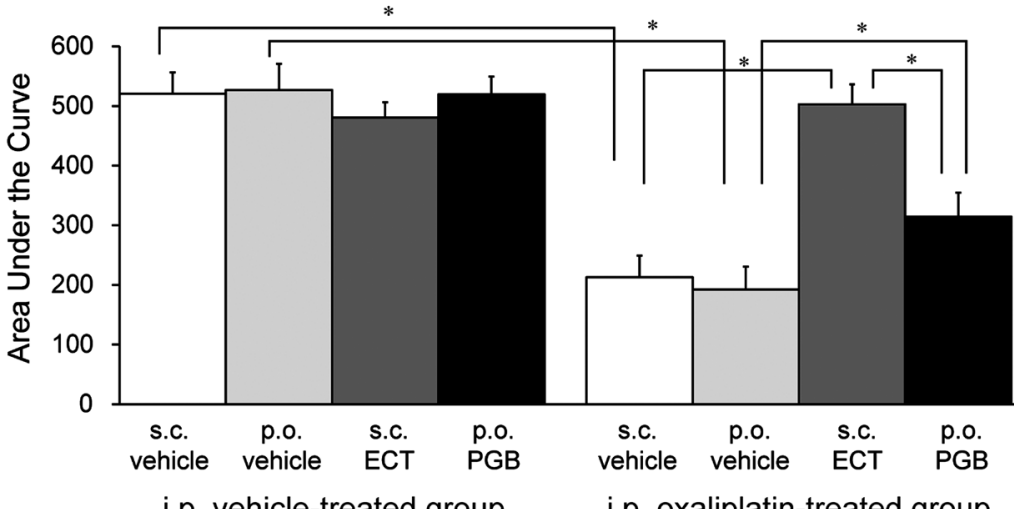

i.p. vehicle-treated group

i.p. oxaliplatin-treated group

Fig. 1. The Effects of Elcatonin or Pregabalin on Oxaliplatin-Induced Cold Allodynia in Rats

The time course of rat hind paw withdrawal latency is indicated for all study groups before and after either (A) elcatonin (ECT) or (B) pregabalin (PGB) administration at the times indicated. Withdrawal latencies represent the mean time \pm S.E.M. It took the rats to withdraw their hind paws from cold stimulation $\left(8^{\circ} \mathrm{C}\right) .{ }^{*} p<0.05, * * p<0.01$, compared to the i.p vehicle plus s.c. vehicle or p.o. vehicle group; ${ }^{\dagger} p<0.05,{ }^{\dagger \dagger} p<0.01$, compared to the i.p. vehicle plus s.c. ECT or $p . o$. PGB group; ${ }^{\#} p<0.05$, ${ }^{\#} p<0.01$, compared to the i.p. oxaliplatin plus s.c. ECT or p.o. PGB group, as appropriate. The mean areas under these hind paw withdrawal latency versus time curves (AUC) were calculated using the trapezoidal rule $(\mathrm{C}) .{ }^{*} p<0.05$, for the comparisons indicated.

served that the pregabalin-treated rats often yawned and shut their eyes. A previous study reported that pegabalin $(30 \mathrm{mg} /$ $\mathrm{kg}$ )-injected rats appeared to be suffering from severe somnolence or dizziness. ${ }^{11)}$ The yawning and eye closure behaviors may be due to sedative effects of pregabalin, because somno- lence is known to be the major side effects of pregabalin. ${ }^{4,5)}$

In a previous study, the anit-allodynic effects of morphine, pregabalin and lidocaine was evaluated by calculating the mean area under the withdrawal latency versus time curve $(A U C)$ using the trapezoidal rule. They compared the calcu- 

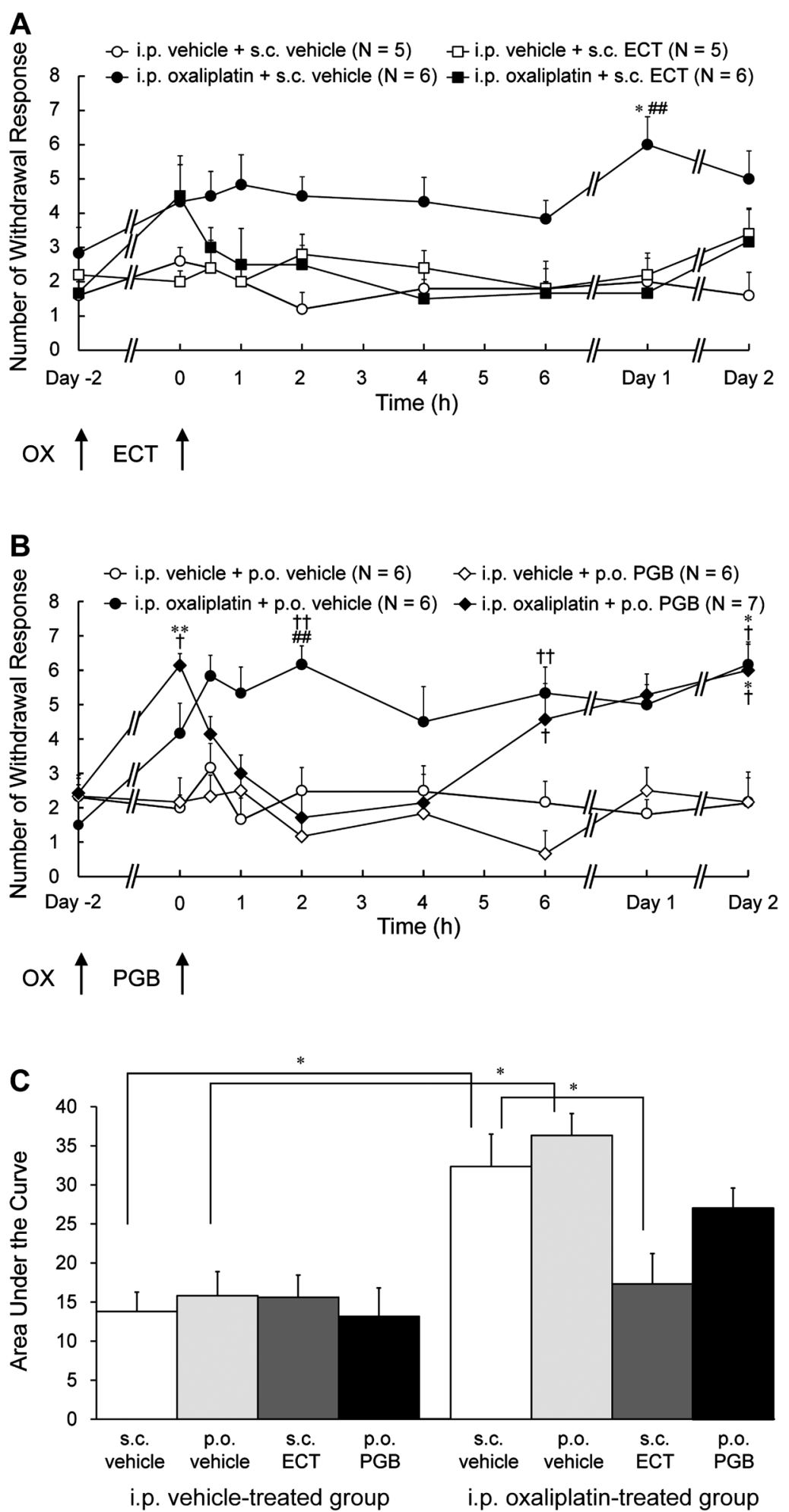

Fig. 2. The Effects of Elcatonin or Pregabalin on Oxaliplatin-Induced Mechanical Allodynia in Rats

The time course of rat hind paw withdrawal using the von Frey test is indicated for all study groups before and after either (A) elcatonin (ECT) or (B) pregabalin (PGB) administration at the time indicated. The $y$-axis indicates the mean number \pm S.E.M. of hind paw withdrawals evoked on testing with the $4 \mathrm{~g}$ von Frey filament. $* p<0.05$, ${ }_{* *}^{*} p<0.01$, compared to the i.p vehicle plus s.c. vehicle or $p . o$. vehicle group; ${ }^{\dagger} p<0.05,{ }^{\dagger} p<0.01$, compared to the compared to the i.p. vehicle plus $p . o$. PGB group; $\# p<0.01$, compared to the i.p. oxaliplatin plus s.c. ECT or p.o. PGB group, as appropriate. The mean areas under these hind paw withdrawal responses versus time curves $(A U C)$ were calculated using the trapezoidal rule $(\mathrm{C}) .{ }^{*} p<0.05$ for the comparisons indicated.

lated $A U C$ s using one-way ANOVA followed by post-hoc test, regardless of the presence or absence of statistical significance among the vehicle-injected group and the drug-treated groups at each time point. ${ }^{3)}$ We evaluated the anti-allodynic effect of elcatonin and pregabalin by calculating the $A U C$, accord- ing to the report. Comparison of the effects indicated that the anti-allodynic effects of elcatonin $(20 \mathrm{U} / \mathrm{kg}$, s.c.) were greater than the effects of pregabalin $(30 \mathrm{mg} / \mathrm{kg}$, p.o. $)$. The results of the present study therefore suggested that elcatonin showed a performance advantage, by producing more sustained anti- 
allodynic effects without obvious signs of somnolence.

Although the plasma half-life of elcatonin is approximately $40 \mathrm{~min}$, a single administration of elcatonin reduced oxaliplatin-induced neuropathy from $0.5 \mathrm{~h}$ after administration until Day 2 of the study. In our previous work, we found that repeated administration of elcatonin resulted in the persistence of its effects for $3 \mathrm{~d}$ after the cessation of treatment. ${ }^{6)}$ These facts suggest that changes in gene expression may be involved in the anti-allodynic effects of elcatonin. Further studies were needed to clarify the mechanism of the action of elcatonin.

In conclusion, to our knowledge, the present study first showed that a single administration of elcatonin reversed the effects of both cold and mechanical allodynia induced by oxaliplatin in rats for a longer time period than pregabalin does. The results of the present study could strengthen an opportunity of elcatonin for clinical treatment of oxaliplatin-induced neuropathy.

Acknowledgment Elcatonin and pregabalin were kindly gifted by Asahi Kasei Pharma Corporation (Tokyo, Japan).

\section{REFERENCES}

1) Baker DE. Oxaliplatin: a new drug for the treatment of metastatic carcinoma of the colon or rectum. Rev. Gastroenterol. Disord., 3, 31-38 (2003).

2) Pasetto LM, D’Andrea MR, Rossi E, Monfardini S. Oxaliplatinrelated neurotoxicity: how and why? Crit. Rev. Oncol. Hematol., 59, 159-168 (2006)

3) Ling B, Coudore F, Decalonne L, Eschalier A, Authier N. Comparative antiallodynic activity of morphine, pregabalin and lidocaine in a rat model of neuropathic pain produced by one oxaliplatin injection. Neuropharmacology, 55, 724-728 (2008).

4) Saif MW, Syrigos K, Kaley K, Isufi I. Role of pregabalin in treatment of oxaliplatin-induced sensory neuropathy. Anticancer Res., 30, 2927-2933 (2010).

5) Satoh J, Yagihashi S, Baba M, Suzuki M, Arakawa A, Yoshiyama T, Shoji S. Efficacy and safety of pregabalin for treating neuropathic pain associated with diabetic peripheral neuropathy: a 14 week, randomized, double-blind, placebo-controlled trial. Diabet. Med., 28, 109-116 (2011).

6) Aoki M, Mori A, Nakahara T, Sakamoto K, Ishii K. Effect of synthetic eel calcitonin, elcatonin, on cold and mechanical allodynia induced by oxaliplatin and paclitaxel in rats. Eur. J. Pharmacol., 696, 62-69 (2012).

7) Aoki M, Mori A, Nakahara T, Sakamoto K, Ishii K. Salmon calcitonin reduces oxaliplatin-induced cold and mechanical allodynia in rats. Biol. Pharm. Bull., 36, 326-329 (2013).

8) Field MJ, Bramwell S, Hughes J, Singh L. Detection of static and dynamic components of mechanical allodynia in rat models of neuropathic pain: are they signalled by distinct primary sensory neurones? Pain, 83, 303-311 (1999).

9) Peng P, Xi Q, Xia S, Zhuang L, Gui Q, Chen Y, Huang Y, Zou M, Rao J, Yu S. Pregabalin attenuates docetaxel-induced neuropathy in rats. J. Huazhong Univ. Sci. Technolog. Med. Sci., 32, 586-590 (2012).

10) Takayanagi N, Shibata K, Ohno H, Nakagami K, Shinoda Y, Kuroda K, Hayano K, Shimizu T, Miyazaki M, Toda N, Tsujimoto A. General pharmacological properties of elcatonin. Prog. Med. (Tokyo), 2, 63-77 (1982).

11) Muratake $H$, Ito $A$, Toda $T$, Suzuki $H$, Fukasawa $H$, Tsuda M, Inoue K, Sugiyama K, Shudo K. $(R)$ - and $(S)$-4-Amino-3-(trimethylsilyl)methylbutanoic acids ameliorate neuropathic pain without central nervous system-related side effects. Bioorg. Med. Chem. Lett., 22, 7602-7604 (2012). 